Uso de la metodología de "Aprendizaje Basado en Equipos (ABE)". Contribuciones al logro de un enfoque de aprendizaje profundo y a la mejora de la enseñanza

\author{
Romina Gómez, Eladio Donoso
}

Enviado: $29 / 11 / 2017$

Aceptado: 23/03/2018

Publicado en prensa: 04/06/2018

Publicado: 04/07/2018

\title{
//Resumen
}

INTRODUCCIÓN. El Aprendizaje Basado en Equipos es una metodología que ha sido utilizada con éxito en educación superior. Esta facilita el aprendizaje activo de los estudiantes y la diversificación de la enseñanza de los docentes. MÉTODO. En este contexto, este artículo presenta una experiencia en la cual la metodología Aprendizaje Basado en Equipos se aplica a un grupo numeroso de estudiantes que cursan una asignatura de una carrera universitaria para evaluar su efectividad, tanto en la enseñanza, como en el logro de aprendizajes profundos. RESULTADOS. Los resultados obtenidos dan cuenta de que esta metodología, por un lado, propició el aprendizaje profundo, puesto que el $68 \%$ de los estudiantes abordó la asignatura, según Study Process Questionnaire, desde un enfoque profundo de aprendizaje y, por otro lado, promovió experiencias favorables de aprendizaje, lo que se evidencia en el índice general de las cuatro dimensiones consideradas para su evaluación (buena enseñanza, metas claras, carga de trabajo y evaluación apropiada), el cual fue de 74 en un índice del 1 al 100. DISCUSIÓN. Los hallazgos se discuten sobre la base del modelo de Presagio, Proceso y Producto para medir la efectividad de la metodología empleada.

\section{//Palabras clave}

Aprendizaje Basado en Equipos; Enfoques de aprendizaje; Experiencias de innovación en educación superior; Educación universitaria.

\section{//Referencia recomendada}

Gómez, R., y Donoso, E. (2018). Uso de la metodología de "Aprendizaje Basado en Equipos (ABE)". Contribuciones al logro de un enfoque de aprendizaje profundo y a la mejora de la enseñanza. REIRE Revista d'Innovació i Recerca en Educació, 11(2), 31-44. http://doi.org/10.1344/reire2018.11.220603

//Datos de los autores

Romina Gómez. Universidad Católica del Norte, Chile. rgomez02@ucn.cl, ORCID: http://orcid.org/0000-0002$\underline{5162-8477}$

Eladio Donoso. Universidad Católica del Norte, Chile. edonoso@ucn.cl, ORCID: http://orcid.org/0000-00028961-2489 
//Títol

Ús de la metodologia de l'aprenentatge basat en equips (ABE). Contribucions a l'assoliment d'un enfocament d'aprenentatge profund i a la millora de l'ensenyament

\section{//Resum}

INTRODUCCIÓ. L'aprenentatge basat en equips és una metodologia que ha estat utilitzada amb èxit en l'educació superior. Facilita l'aprenentatge actiu dels estudiants i la diversificació de l'ensenyament dels docents. MÈTODE. En aquest context, aquest article presenta una experiència en la qual la metodologia de l'aprenentatge basat en equips s'aplica a un grup nombrós d'estudiants que cursen una assignatura d'una carrera universitària, per avaluar-ne l'efectivitat tant en l'ensenyament com en l'assoliment d'aprenentatges profunds. RESULTATS. Els resultats obtinguts mostren, d'una banda, que aquesta metodologia va propiciar l'aprenentatge profund, ja que el $68 \%$ dels estudiants va abordar l'assignatura, d'acord amb /'Study Process Questionnaire, des d'un enfocament profund d'aprenentatge; d'altra banda, mostren que va promoure experiències favorables d'aprenentatge, la qual cosa s'evidencia en l'índex general de les quatre dimensions considerades per a la seva avaluació (bon ensenyament, metes clares, càrrega de treball i avaluació apropiada), el qual va ser de 74 en un índex de I'1 al 100. DISCUSSIÓ. Les troballes es discuteixen sobre la base del model de presagi, procés i producte per mesurar l'efectivitat de la metodologia emprada.

\section{//Paraules clau}

Aprenentatge basat en equips; Enfocaments d'aprenentatge; Experiències d'innovació en educació superior; Educació universitària.

\section{//Title}

Using team-based learning: Contributions to the achievement of a deep learning approach and the improvement of teaching

\section{//Abstract}

INTRODUCTION. Team-based learning provides a methodology that has been successfully used in higher education. The methodology facilitates students' active learning and allows teachers to diversify their teaching practice. METHOD. The article describes an experience in which team-based learning methodology was applied to a large group of students enrolled in a university course and evaluates its effectiveness in supporting both teaching and deep learning. RESULTS. The methodology was shown to foster deep learning insofar as $68 \%$ of the students undertook the course adopting a deep learning approach, according to the Study Process Questionnaire applied. The methodology also promoted favourable learning experiences, as evidenced in the results of the general index of the four dimensions used to evaluate the TBL approach (good teaching, clear goals, workload and appropriate evaluation), which gave a score of 74 in an index of 1 to 100 . DISCUSSION. The findings are discussed using the presage, process, product (3P) model to measure the effectiveness of the methodology.

\section{//Keywords}

Team-based learning; Learning approaches; Experiences of innovation in higher education; University education.

Aquest treball està publicat amb llicència Creative Commons Attribution. Per veure una còpia de la llicència, visiteu https://creativecommons.org/licenses/by/4.0/ Esta obra está publicada bajo la Licencia de Atribución de Creative Commons. Para ver una copia de la licencia, visite https://creativecommons.org/licenses/by/4.0/ This work is licensed under the Creative Commons Attribution License. To view a copy of the license, visit https://creativecommons.org/licenses/by/4.0/ 


\section{Introducción}

En las últimas décadas se ha dado particular énfasis a los procesos de enseñanza y aprendizaje en educación superior (Biggs y Tang, 2007; Bowden y Marton, 2004; Ellis y Goodyear, 2010; González, Montenegro, López, Munita y Collao, 2011; Prosser y Trigwell, 1999). Dichos procesos se han centrado especialmente en los enfoques y aproximaciones metodológicas respecto al aprendizaje 'superficial' y al aprendizaje 'profundo' (Haggis, 2009). Lo anterior se relaciona estrechamente con la calidad de los aprendizajes a nivel universitario, fundamentalmente en contextos donde se presenta el fenómeno de matrículas masivas en las universidades, lo que conlleva la creación de cursos numerosos. Ante este escenario, se hace necesaria la implementación de metodologías que salvaguarden dicha calidad.

Considerando lo planteado, el Aprendizaje Basado en Equipos (en adelante, ABE) surge como una alternativa interesante, puesto que promueve el uso de habilidades cognitivas superiores (aprendizaje profundo) y experiencias de aprendizaje favorables en cursos numerosos. Esto es así ya que contempla diversos escenarios didácticos con énfasis en el trabajo entre pares, en donde el rol del profesor es ser un facilitador del proceso de enseñanza y aprendizaje (Lee, Lagos y Mella, 2014).

En el contexto chileno, a partir de la década de los 90 , se ha incrementado la matrícula de estudiantes en las universidades, lo que ha repercutido en un incremento en el número de estudiantes por curso. No obstante, las instituciones de educación superior chilenas no claudican en promover que el docente oriente su enseñanza hacia el uso de metodologías activas que aseguren la calidad del proceso educativo. Para este fin, se deben considerar los múltiples factores que se movilizan dentro del aula, como, por ejemplo, las características de los estudiantes, aspectos socioculturales, las propias creencias pedagógicas, o el número de estudiantes por curso, entre otros (Ginns, Kitay y Prosser, 2008; González, 2010, 2011; González et al., 2011; Sobrados, 2016).

Lo anterior no es una tarea fácil, si se considera que la mayoría de las propuestas sobre metodologías activas recomiendan trabajar con un máximo de 25 estudiantes en el aula (Sobrados, 2016). En Chile, como ya se ha mencionado, la cantidad de estudiantes por clase es alta, sobrepasando lo que se estima como ideal. De este modo, se observa que, frente a esto, el docente universitario opta por seguir empleando la clase magistral como estrategia de enseñanza. Ante este panorama, no se debe perder de vista que es el estudiante el agente más importante dentro del aula, siendo el protagonista y beneficiario del aprendizaje en interacción con sus pares (Biggs, 2008).

En atención a lo expuesto, en este artículo se presenta una experiencia pedagógica cuyo objetivo es implementar la metodología $A B E$ en un curso numeroso de nivel universitario, con el fin de evaluar su efectividad como recurso de enseñanza-aprendizaje. Dicha efectividad se mide a través de los cuestionarios Study Process Questionnaire y Course Experience Questionnaire (Marchant, Fauré y Abricot, 2016). 


\section{Aprendizaje basado en equipos}

Un elemento clave de la metodología $A B E$ es que permite al docente diversificar la enseñanza, puesto que, más allá de simplemente cubrir contenidos, se busca asegurar que los estudiantes tengan la posibilidad y la oportunidad de usar conceptos para resolver problemas que se les planteen en la clase y que, probablemente, enfrentarán en su desempeño profesional en el futuro. De esta forma, a los estudiantes se les provee de conocimiento conceptual y procedimental (Michaelsen y Sweet, 2008), convirtiéndose en una alternativa para que desarrollen habilidades cognitivas y actitudinales, tales como el aprendizaje profundo, la motivación y el trabajo en equipo (Lee et al., 2014).

La metodología del $A B E$ está fundamentada en cuatro principios que son indispensables para asegurar su éxito. Estos principios, según Michaelsen y Sweet (2008), son:

- Equipos formados estratégicamente. Estos deben ser heterogéneos y permanentes durante el curso, con un número de entre cinco y siete integrantes.

- Responsabilidad individual por parte de los estudiantes para preparar las lecturas pre-clase y contribuir al éxito del equipo.

- Actividades en clase que obligan a los estudiantes a tomar decisiones, las cuales deben estar relacionadas con los resultados de aprendizaje y ser reportadas en forma precisa.

- Retroalimentación frecuente y oportuna por parte del profesor hacia los alumnos.

Ahora bien, el $A B E$ se caracteriza por contar con tres fases, las cuales se establecen para una unidad de contenido. La figura 1 muestra la secuencia de las tres fases de ABE.

Figura 1

Secuencia de las fases del ABE

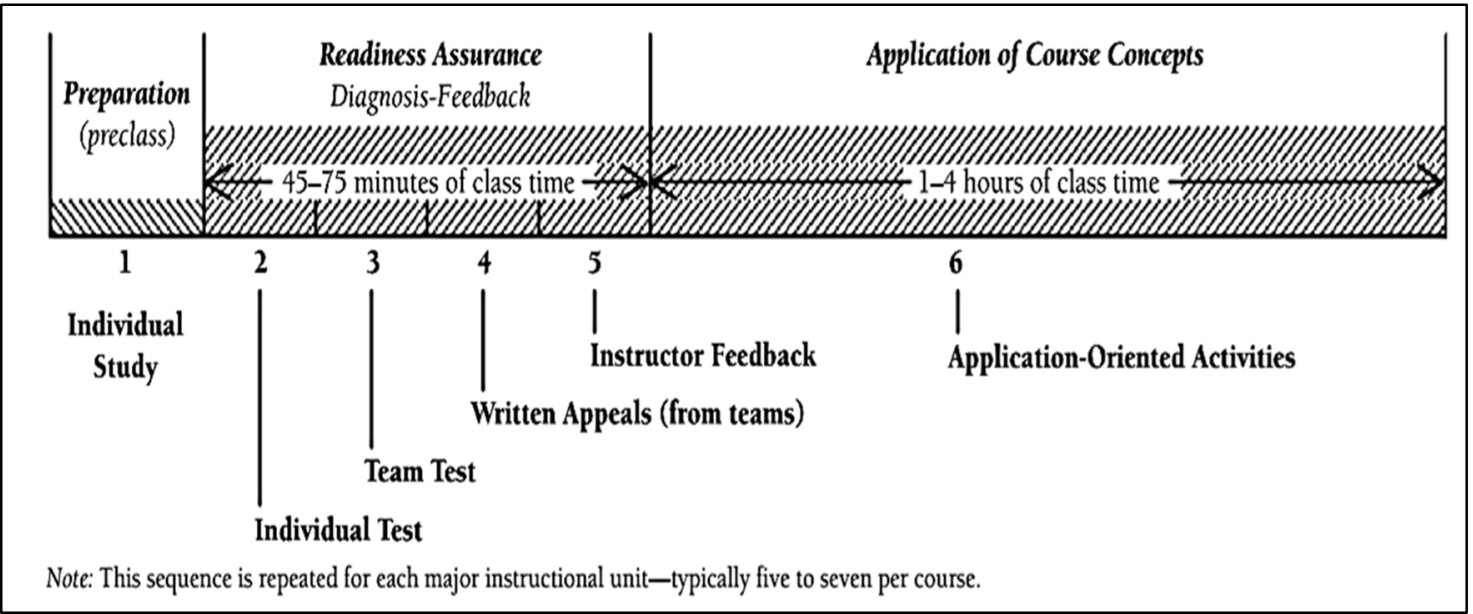

Fuente: Michaelsen y Sweet (2008. p. 9).

En la fase 1 el estudiante realiza un trabajo o estudio individual pre-clase. Esto implica la preparación previa del material por parte del docente, es decir, debe preparar las lecturas y formular preguntas en formato de selección múltiple. La fase 2, para los estudiantes, contempla 
responder una prueba individual y, luego, una discusión grupal de los resultados individuales de las pruebas. En esta fase cada grupo puede dar argumentos por escrito de sus respuestas que aún considera correctas. Una vez hecho lo anterior, se realiza una discusión en clase. Aquí, el profesor participa de la discusión con la clase y provee retroalimentación. La fase 3, por su parte, consiste en la explicación de conceptos clave y la asignación de tareas por parte del docente. En este punto se espera que los estudiantes apliquen dichos conceptos de manera que den cuenta, tanto al profesor como a sus compañeros de equipo, de la cantidad y la calidad de su trabajo individual (Ruiz-Campo, Soria-Barreto y Zuñiga-Jara, 2016).

\section{Descripción de la experiencia}

\section{Contexto de la experiencia}

Esta experiencia pedagógica se enmarca en la carrera de Psicología de una universidad del norte de Chile, específicamente en el curso denominado Psicología Organizacional, que se imparte en el cuarto semestre académico del programa de estudios con cinco unidades del Sistema de Créditos Transferibles (SCT) equivalentes a cuatro horas y media de docencia directa (clases) y tres horas de trabajo autónomo (fuera de clases), con un total semanal de siete horas y media cronológicas de dedicación.

Los resultados de aprendizaje de este curso apuntan a que los estudiantes, por una parte, comprendan elementos centrales de la psicología organizacional y, por otra, analicen el comportamiento organizacional con capacidad crítica.

Los participantes de la experiencia fueron 37 estudiantes, cuyo promedio alcanzado en la prueba de selección universitaria fue de 578, de un máximo esperado de 800 puntos. Por otra parte, el $42,6 \%$ provienen de establecimientos semiprivados y el $29,3 \%$ de establecimientos públicos. Esto es considerablemente importante, puesto que, en Chile, este tipo de establecimientos no se caracteriza por su excelencia académica, lo que se condice con el puntaje promedio de ingreso en la prueba de selección. Con respecto a sus hábitos de estudios, los antecedentes recogidos por instrumentos internos de la carrera informan que, en general $(57,6 \%)$, no presentan un horario de estudio, siendo el máximo de tiempo destinado a esta actividad seis horas a la semana (66,7\%). En relación con sus habilidades cognitivas, poseen un nivel de razonamiento concreto $(60,8 \%)$, es decir, las operaciones mentales que realizan requieren de experiencias concretas.

\section{Sistematización de la experiencia}

Esta experiencia pedagógica se inició con la conformación de los equipos de trabajo de cinco integrantes como máximo, designados de manera aleatoria por el docente al inicio del semestre académico, cautelando las condiciones de heterogeneidad del equipo, tales como rendimiento académico, género y edad. Esta conformación de equipos se mantuvo estable durante el semestre académico en el cual se llevó a cabo la experiencia.

Para realizar la fase 1 para cada sesión, esto es la preparación para el aprendizaje, se diseñaron actividades como lecturas, videos y foro-debates. Al ser actividades desarrolladas 
individualmente durante las tres horas de trabajo autónomo, se utilizó la plataforma virtual institucional. De esta manera, el estudiante podía ingresar a dicha plataforma de acuerdo con su organización de tiempo.

La fase 2 se realizó durante los noventa minutos que duraba cada sesión de clase. Durante los primeros treinta minutos, se usó una prueba individual para medir el manejo de conocimientos, con diez preguntas de selección múltiple. Luego, en los siguientes treinta minutos, los estudiantes se organizaron en equipos para realizar el mismo control previo, pero ahora grupalmente. Aquí, el equipo consensuaba y decidía las respuestas finalmente correctas. Se esperaba que los estudiantes pudieran discutir en relación con sus respuestas individuales, pero, esta vez, confrontándolas con las de sus compañeros de equipo. Es decir, a partir de la prueba individual, los estudiantes reconocían lo que no entendían y, luego, en el trabajo entre pares, discutían, analizaban y construían argumentos lógicos para, así, elegir la respuesta correcta.

Para evaluar el trabajo realizado por los equipos en la fase 2, se les aplicó, inmediatamente terminada la actividad, la tarjeta IF-AT (Figura 2), por sus siglas en inglés Immediate Feedback Assessment Technique (Epstein et al., citado en Apple, 2013). Esta tarjeta muestra efectividad, debido a que los estudiantes obtienen de manera rápida información sobre las respuestas. De esta manera, se estimula que los estudiantes argumenten, debatan y modifiquen sus aprendizajes de forma inmediata. Esto, a su vez, facilita los aprendizajes de tipo profundo y le permite al docente conocer aquellos ámbitos en que los estudiantes presentan falencias, optimizando el proceso de enseñanza y aprendizaje.

Esta tarjeta se caracteriza por tener un formato de respuesta de selección múltiple, con opciones de raspado. Si la respuesta es correcta, aparecerá un símbolo (estrella) y en caso contrario los estudiantes pueden hacer otro intento de respuesta. Pues bien, su aplicación permitió que los mismos estudiantes cotejaran sus aciertos y errores y se generara una discusión inmediata al interior de cada equipo.

Figura 2

Tarjeta IF-AT

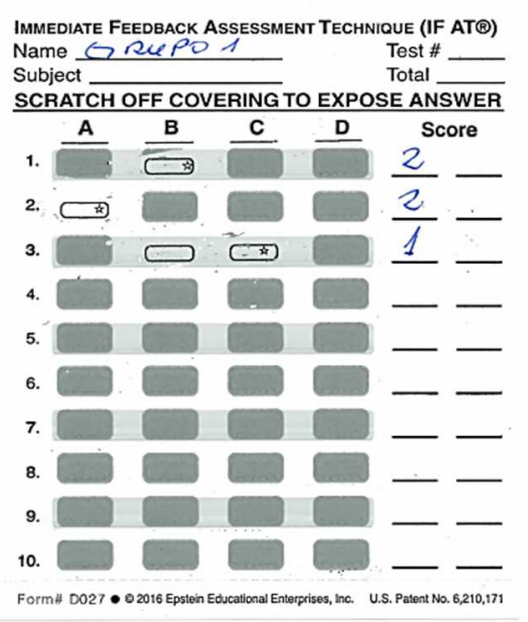

Fuente: Elaboración propia. 
Finalmente, durante la fase 3, denominada aplicación, se optó por trabajar con la resolución de problemas a través de casos durante noventa minutos de clases. A través del desarrollo de casos se promovió que los estudiantes desarrollaran habilidades como pensamiento crítico, capacidad de análisis, toma de decisiones, entre otras. El contenido de estos casos fueron situaciones verídicas del ámbito de la psicología organizacional, cuyos temas fueron tratados en las fases previas. Para el desarrollo del caso, los estudiantes debieron leer y reflexionar en sus equipos $y$, luego, responder varias preguntas de información, tales como: ¿de qué manera el psicólogo puede contribuir a la resolución de esta problemática?, ¿qué aspectos de la teoría se pueden utilizar como estrategias para solucionar el problema?, o ¿cómo el protagonista del caso puede optimizar recursos?

Cabe destacar que el caso fue el mismo para todos los equipos. Esto facilitó que focalizaran su atención en las conclusiones, reflexiones y sugerencias de los otros grupos, generándose la retroalimentación.

Es importante señalar que, durante las tres fases, el profesor generó retroalimentación de los aspectos más relevantes a estudiar, ofreciendo documentación y bibliografía por medio de la plataforma virtual, facilitando la organización del tiempo de estudio y el trabajo semanal a realizar. Esto fue cíclico durante el semestre.

De manera complementaria, se realizaron visitas a organizaciones, planificadas como actividad en terreno, cuyo objetivo fue que los estudiantes lograran transferir los conceptos estudiados a actuales escenarios de desarrollo de la psicología organizacional.

Para cautelar que el proceso evaluativo fuese acorde a las competencias de análisis crítico que esta asignatura pretendía movilizar, se consideró que los instrumentos utilizados fueran de aplicación, acompañados de una rúbrica analítica que establecía los criterios a considerar durante el proceso. Es importante indicar que los estudiantes tuvieron acceso a la rúbrica con antelación y que, una vez realizado el proceso, el docente generó espacios de retroalimentación.

\section{Evaluación del proceso enseñanza-aprendizaje}

Para evaluar el proceso de enseñanza y aprendizaje, acorde a la metodología empleada en esta experiencia, se utilizó el Study Process Questionnaire (Marchant et al., 2016), que consiste en dos escalas. La primera es para medir el aprendizaje superficial, la que contiene afirmaciones tales como: mi propósito es aprobar este curso haciendo el menor esfuerzo posible; aprendo algunas cosas de este curso repasándolas una y otra vez hasta que las sé de memoria, aunque no las comprenda. Por su parte, la segunda escala es para evaluar aprendizaje profundo, algunas de sus afirmaciones son: cuando estudio un tema de este curso, no estoy conforme hasta que le dedico tiempo suficiente para formar mis propias conclusiones; frecuentemente paso tiempo extra buscando información adicional sobre temas de este curso, ya que me parecen interesantes.

También se utilizó el cuestionario Course Experience Questionnaire (Marchant et al., 2016) compuesto por cuatro dimensiones: buena enseñanza, metas claras, carga de trabajo y evaluación apropiada. 
La dimensión buena enseñanza contempla afirmaciones tales como: el profesor de este curso me motiva a hacer mi mejor trabajo; el profesor se esfuerza para que la materia sea interesante. Para el caso de la dimensión metas claras. el profesor dejó en claro, desde el inicio del curso, lo que se esperaba de los estudiantes; usualmente he tenido una idea clara de lo que se espera de mí en este curso. Ejemplos de afirmaciones asociadas a la dimensión carga de trabajo son: en este curso se otorga suficiente tiempo para comprender los contenidos propuestos; la carga de trabajo en este curso es muy pesada. Por último, para la dimensión evaluación de los aprendizajes podemos ilustrar lo siguiente: solo se necesita buena memoria para que a uno le vaya bien en este curso; el profesor está más interesado en evaluar lo que he memorizado que lo que he comprendido (Marchant et al., 2016) adaptado de Entwistle y Ramdsen (1983). Resulta relevante señalar que ambos cuestionarios han sido adaptados al contexto chileno (Marchant et al., 2016).

Finalmente, una encuesta de satisfacción de corte cualitativo, que explora en temáticas como metodologías, evaluación y propuesta de mejoras, complementó la recolección de información.

\section{Resultados}

Habiendo aplicado durante todo el semestre académico la metodología $A B E$, los resultados obtenidos dan cuenta de que esta experiencia pedagógica propició, para estos estudiantes, el uso de habilidades cognitivas superiores (aprendizaje profundo) y, a su vez, se percibió como favorable en el contexto de cursos numerosos.

Con respecto a los enfoques de aprendizaje, que predominan entre el grupo de 37 estudiantes, 25 de ellos utilizaron enfoque profundo y 12 enfoque superficial. Es decir, el $68 \%$ de los estudiantes utilizaron estrategias de orden superior para elaborar sus aprendizajes en este curso.

En relación con las experiencias de aprendizaje, el índice general de las cuatro dimensiones fue de 74 en un índice del 1 al 100'. En específico por escala de cada dimensión, evaluación apropiada tuvo una valoración de 78, buena enseñanza y metas claras de 74 y carga de trabajo tuvo una valoración de 70 .

Considerando el índice general (74), podemos señalar que los estudiantes estimaron favorablemente la experiencia de aprendizaje. En referencia a la escala mejor valorada, evaluación apropiada (78), se plantea que los estudiantes percibieron que las estrategias evaluativas apuntaron a evaluar lo comprendido y no lo memorizado. Asimismo, cuando se les consultó por las estrategias evaluativas, se señala:

las evaluaciones siempre fueron completamente ligadas a lo explicado en clases, en otras asignaturas en ocasiones las evaluaciones no van $100 \%$ ligado a lo expuesto por el docente a diferencia de esta asignatura, donde reflejar lo explicado era sencillo debido a las clases [E2].

Por una parte, esto se explica porque a diferencia de la cohorte anterior, se utilizaron evaluaciones en directa relación con lo tratado en clases. En el ABE se espera que los estudiantes

\footnotetext{
1 Se considera favorable sobre un índice 50 (Alba y Schumacher, 2008).
} 
aprendan de sus errores, los rectifiquen y logren generar análisis de manera más continua. Además, el $A B E$ propicia que las evaluaciones tengan una mayor frecuencia que bajo el modelo tradicional.

Respecto a la valoración de la escala buena enseñanza (74), podemos indicar que los estudiantes apreciaron la metodología aplicada, tanto por la orientación de enseñanza centrada en los aprendizajes, como por la motivación hacia la asignatura, el trabajo en equipo y la grata experiencia de aprendizaje. A este respecto, los estudiantes manifestaron, por ejemplo, lo siguiente:

las metodologías activas utilizadas en la asignatura facilitaron mi aprendizaje, pues me motivaban y ayudaban a mantener la concentración en las clases, además de promover la participación en la misma, y generar una grata experiencia de aprendizaje colectivo" [E1]. Complementariamente, para el uso del raspe se plantea: "para los raspes, la utilización del modelo de 'quién quiere ser millonario', con comodines de llamar a un amigo, 50 y 50 haría aún más lúdica las actividades de aprendizaje [E1].

Por su parte, en lo relacionado con las metas claras (74), los estudiantes reconocen que la asignatura se llevó a cabo considerando la dinámica del ABE en las clases. Al respecto señalaron:

aspectos positivos que rescato de esas metodologías es que siempre fueron en grupo (para una ayuda y trabajo en equipo), ocupaban los tiempos de clases y las retroalimentaciones eran en conjunto con todos haciendo visualizar el error de manera colectiva y resolverlo de la misma forma [E3].

Asimismo, valoraron el cumplimiento de aspectos formales tal como: "siempre se respetaron las fechas, las entregas fueron siempre el día designado y las evaluaciones tuvieron relación con el contenido entregado" [E3]

Ante la dimensión carga de trabajo, con buena valoración (70), pero la menor de las cuatro escalas, la percepción de los estudiantes es que el método aplicado les permite distribuir de mejor forma el tiempo que destinan a sus aprendizajes, pueden preparar mejor el material, logrando comprenderlo con mayor profundidad, a diferencia de la metodología tradicional que implica preparar grandes cantidades de materia en un menor plazo.

Complementariamente, para verificar la relación entre enfoques y experiencias de aprendizaje se empleó el modelo 3P, denominado así puesto que en él se identifican tres etapas: el presagio, antes de que se produzca el aprendizaje; el proceso durante el aprendizaje, y finalmente el producto del aprendizaje (Biggs, 1987; Prosser y Trigwell, 2006). Estos factores interactúan entre sí, condicionando las percepciones y enfoques asumidos por estudiantes y docentes. En términos generales, se plantea que los aprendizajes de los estudiantes responden a la percepción de elementos del contexto, como la buena docencia, las metas claras y la evaluación apropiada, entre otros (Ginns y Ellis, 2009; Webster, Chan, Prosser y Watkins, 2009).

Pues bien, se comparó al grupo de estudiantes que utilizaron estrategias profundas con el grupo de estudiantes que utilizaron estrategias superficiales pertenecientes al mismo curso. Para ello se empleó el estadístico $t$ de Student, en el programa SPSS, versión 21, encontrándose diferencias estadísticamente significativas a favor del grupo aprendizaje profundo en 2,5 puntos, 
específicamente con la dimensión buena enseñanza $[p 0.031<0.5]$, apreciándose que la buena docencia se relaciona con el aprendizaje profundo, como lo señalan González et al. (2011)

Otro planteamiento teórico del modelo 3P, verificado a partir de esta experiencia pedagógica, fue la relación entre la experiencia de aprendizaje favorable y el rendimiento académico. Para ello se contrastaron las medias a través del estadístico $t$ para muestras pareadas empleando el programa STATA versión 14, encontrándose relación al 95\% de significancia. Esta información adquiere sentido, si consideramos que, en la cohorte anterior a esta implementación el rendimiento académico fue menor en 1.2 puntos al rendimiento general del curso con metodologías activas. En relación con el porcentaje histórico de aprobación-reprobación de la asignatura, se puede señalar que el porcentaje de reprobación disminuyó de un 21,5\% a un $1,3 \%$. Esto resulta interesante ya que esta diferencia reflejaría que, a nivel global, bajo la nueva metodología, se promueve un mayor logro de los resultados de aprendizaje de la asignatura.

\section{Discusión y conclusiones}

Este trabajo entrega una mirada general respecto a una experiencia pedagógica que tuvo como objetivo implementar la metodología $A B E$ en un curso numeroso de nivel universitario con el fin de evaluar su efectividad como recurso de enseñanza-aprendizaje. Esto se enmarcó en el curso Psicología Organizacional de una universidad del norte de Chile.

El origen de esta experiencia pedagógica estuvo dado por la complejidad de la asignatura, las características de los estudiantes y el actual escenario universitario. Al respecto, el primer tema relevante que se valoró fue la revisión de modelos explicativos que consideren la multicausalidad del proceso de enseñanza y aprendizaje, optando por modelo 3P.

Para dar cuenta de esta multicausalidad es necesario explicitar que el curso Psicología Organizacional contó con treinta y siete estudiantes, cuyos resultados de aprendizajes apuntan al desarrollo de la capacidad crítica en temáticas de la disciplina. Por otro lado, debemos recordar que las competencias de entrada de los estudiantes no parecen ser las apropiadas para el ingreso a la educación superior. Así las cosas, para propiciar el uso de habilidades cognitivas superiores de estos estudiantes fue necesario implementar una metodología con comprobada efectividad al trabajar con grupos grandes, como es el caso del ABE (Lee et al., 2014). Como resultado de lo anterior, se puede reportar que el $68 \%$ de los estudiantes abordaron la asignatura desde un enfoque profundo de aprendizaje. Lo interesante sería, entonces, analizar los factores que pudieron obstaculizar el desarrollo de este enfoque en todos los estudiantes de la asignatura. Para ello, comenzaremos indicando que, en esta metodología, la clase deja de ser magistral y pasa a tener una estructura diferente, siendo el estudiante el centro, lo que implica mayor autonomía y responsabilidad por parte de este.

Ahora bien, si consideramos que en la muestra estudiada los estudiantes tienen escasos hábitos de estudio (seis horas semanales), es razonable pensar que el trabajo durante las horas autónomas no fuese bien utilizado por todos los estudiantes. A esto se suma que la planificación para el uso de las horas de trabajo autónomo no es una práctica habitual de los docentes universitarios chilenos, siendo esto un desafío a nivel nacional (CRUCH, 2015). En relación con este punto, también podemos señalar que se ha observado que la percepción sobre la carga de 
trabajo presenta correlaciones con el enfoque superficial (Marchant et al., 2016). Tomando en cuenta que esta escala fue la de menor valoración, estas consideraciones nos llevan a pensar que, para futuras implementaciones con sistema de créditos transferibles, es necesario indagar sobre la disposición que demuestran tener los estudiantes para organizar su tiempo y adecuarse al estudio semanal requerido en el curso. También sería importante inquirir sobre otros factores de presagio que pudiesen afectar el estudio autónomo, tal como, por ejemplo, la condición de estudiante trabajador.

Otro antecedente que podemos considerar es que el $A B E$ es una metodología en la que las retroalimentaciones del desempeño, generalmente, se realizan en grupo. Si se toma en cuenta que la mayoría de los estudiantes provienen de sistemas escolares de escasa exigencia académica, es posible pensar que las expectativas en relación con el rol del profesor están asociadas a un sistema tradicional de enseñanza. En dicho sistema, el docente es visto como el centro del aprendizaje, quien entrega los contenidos de manera unilateral. Contrariamente, en el ABE son los estudiantes quienes deben ser activos y responsables de sus aprendizajes. Desde el punto de vista pedagógico, una alternativa para subsanar esta situación es, por una parte, generar mayores instancias de diálogo con los estudiantes en relación con su rol en el logro de sus aprendizajes y, por otra, otorgar mayor importancia a la retroalimentación individual. Esto último se refuerza por los hallazgos en torno a la relevancia de la retroalimentación que consideran que este proceso es fundamental para el desarrollo de los enfoques de aprendizaje (Ion, Silva y Cano, 2013).

A pesar de lo anterior, los hallazgos encontrados en esta experiencia apuntan a que la mayoría de los estudiantes de la asignatura la abordaron utilizando estrategias de tipo profundo, es decir, fueron capaces de generar análisis crítico de las temáticas tratadas, en concordancia con los resultados de aprendizaje del curso.

Sobre las experiencias de aprendizaje, estas fueron reportadas como favorables en sus cuatro dimensiones. Además, se verificaron supuestos teóricos desde el modelo de las 3P, encontrándose relaciones entre los enfoques de aprendizaje profundos y la buena enseñanza. En consecuencia, se podría suponer que el $\mathrm{ABE}$ aportó a la mejora de la percepción del alumnado acerca de la facilidad del aprendizaje. Del mismo modo, a través de este, se estimuló la participación activa, lográndose de mejor manera los resultados de aprendizaje propuestos.

Otro hallazgo en la implementación del ABE fue comprobar la relación entre las experiencias de aprendizaje y el rendimiento académico. En este sentido, las investigaciones señalan que el nivel de buen rendimiento estaría relacionado con los factores movilizados en la enseñanza como, por ejemplo, la forma de evaluar (Doménech, Jara y Rosel, 2004) y los hallazgos de esta experiencia pedagógica apuntan en la misma dirección: los estudiantes valoraron las estrategias evaluativas implementadas, relevando que las formas de evaluar en esta asignatura difieren de lo habitual. Es decir, a diferencia de otras asignaturas, en esta los estudiantes tuvieron diversos escenarios evaluativos (individuales-grupales), conocieron los criterios con anticipación y se promovió la entrega inmediata de los resultados.

No obstante los aciertos de esta experiencia, se reconoce como limitación que no es posible establecer relaciones de causalidad a partir de la sistematización de esta experiencia de innovación pedagógica. Sin embargo, los resultados de esta implementación proporcionan 
antecedentes interesantes del uso de la metodología $\mathrm{ABE}$ en grupos numerosos, con trabajo en subgrupos y sin precisar de grandes recursos de infraestructura.

En síntesis, consideramos que la clave para implementar mejoras en docencia universitaria es contemplar la naturaleza multicausal del proceso de enseñanza y aprendizaje, realizando diagnósticos integrales sobre las características de los estudiantes, así como de procesos institucionales de las nuevas formas de enseñar y de los recursos con los que cuenta el docente al momento de generar sus clases.

Finalmente, creemos que es importante propiciar la revisión constante del proceso de enseñanza y aprendizaje por parte del docente a partir de su propia práctica, lo que le permitirá generar propuestas de mejora en beneficio de los aprendizajes de sus estudiantes. Para ello, son las instituciones de educación superior las llamadas a generar estos espacios por medio de programas de formación docente que posibiliten desarrollar competencias pedagógicas de reflexión del actuar docente, tal como lo permiten, por ejemplo, los diplomados en docencia universitaria. Esto posibilitaría que la discusión sobre estas temáticas sobrepase las experiencias particulares y se posicionen en el ámbito de lo institucional.

\section{<Referencias bibliográficas >}

Alba, F., y Schumacher, C. (2008). Evaluación del aprendizaje universitario. Educación y educadores, 11(2), 91-105.

Apple, K. (2013). Embedding assessment into college-level psychology courses. En D. Dun, C. Baker, C. M. Mehrotra, R. E. Landrum, y J. H. Wilson (Eds.), Assessing teaching and learning psychologist (pp. 35-43). Belmont: Waswordth, Cengage Learning.

Biggs, J. (1987). The Study Process Questionnaire (SPQ): Manual. Hawthorn: Australian Council for Educational Research.

Biggs, J. (2008). Calidad del aprendizaje universitario. Madrid: Narcea.

Biggs, J., y Tang, C. (2007). Teaching for quality learning at university: what the student does (3a ed). Philadelphia, Pa.: Society for Research into Higher Education / Open University Press.

Bowden, J., y Marton, F. (2004). The university of learning. London: Routledge.

CRUCH (2015). Informe de gestión. Vicepresidencia ejecutiva del consejo de rectores de universidades chilenas, CRUCH. Período noviembre de 2011 a marzo de 2015. CRUCH.

Doménech, F., Jara, P., y Rosel, J. (2004). Percepción del proceso de enseñanza/aprendizaje desarrollado en Psicoestadística I y su incidencia en el rendimiento. Psicothema, 16, 32-38.

Ellis, R., y Goodyear, P. (2010). Students' experiences of e-learning in higher education. The ecology of sustainable innovation. New York \& London: Routledge.

Entwistle, N. J., y Ramsden, P. (1983). Understanding student learning. London: Croom Helm. 
Ginns, P., y Ellis, R. A. (2009). Evaluating the quality of e-learning at the degree level in the student experience of blended learning. British Journal of Educational Technology, 40(4), 652663. http://doi.org/10.1111/j.1467-8535.2008.00861.x

Ginns, P., Kitay, J., y Prosser, M. (2008). Developing conceptions of teaching and the scolarship of teaching through a graduate certificate in higher education. The international Journal for Academic Development, 13, 175-185. http://doi.org/10.1080/13601440802242382

González, C. (2010). Investigación sobre el aprendizaje y conocimiento académico sobre la enseñanza como claves para mejorar la docencia universitaria. Revista Calidad en la Educación, $33\left(2^{\circ}\right.$ semestre $), 126-143$.

González, C. (2011). Extending research on conceptions of teaching commonalities and differences in recent investigations. Teaching in Higher Education, 16(1), 65-80. http://doi.org/10.1080/13562517.2010.507302

González, C., Montenegro, H., López, L., Munita, I., y Collao, P. (2011). Relación entre la experiencia de aprendizaje de estudiantes universitarios y la docencia de sus profesores. Revista Calidad en la Educación, 35(2 ${ }^{\circ}$ semestre), 21-49. http://doi.org/10.4067/S0718$\underline{45652011000200002}$

Haggis, T. (2009). What Have We Been Thinking of? A Critical Overview of 40 Years of Student Learning Research in Higher Education. Studies in Higher Education, 34(4), 377-390. http://doi.org/10.1080/03075070902771903

Ion, G., Silva, P., y Cano, E. (2013). El feedback y el feedforward en la evaluación de las competencias de estudiantes universitarios. Profesorado. Revista de Curriculum y Formación de Profesorado, 17(2), 283-301.

Lee, X., Lagos, K., y Mella, J. (2014). Formación docente en el aprendizaje activo a través de las técnicas Team Based Learning e Immediate Feedback Assessment Technique. Revista de Educación en Ciencia de la Salud, 11(2), 154-160.

Marchant, J., Fauré, J., y Abricot, N. (2016). Adaptación y validación del SPQ y el CEQ para el estudio de la formación en docencia universitaria en el contexto chileno. Psykhe, 25(2), 1-18. http://doi.org/10.7764/psykhe.25.2.873

Michaelsen, L. K., y Sweet, M. (2008). The essential elements of team-based learning. New directions for teaching and learning, 116, 7-27. http://doi.org/10.1002/tl.330

Prosser, M., y Trigwell, K. (1999). Understanding learning and teaching. The experience in higher education. Buckingham: SRHE y Open University Press.

Prosser, M., y Trigwell, K. (2006). Confirmatory factor analysis of the approaches to teaching

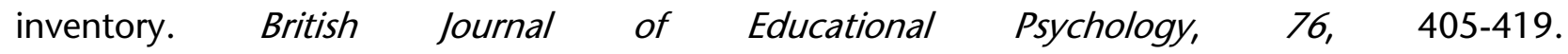
http://doi.org/10.1348/000709905X43571 
Romina Gómez, Eladio Donoso. Uso de la metodología de "Aprendizaje Basado en Equipos (ABE)". Contribuciones al logro de un...

Ruiz-Campo, S., Soria-Barreto, K. y Zuñiga-Jara, S. (2016). Aprendizaje basado en equipos con IFAT: Impacto y percepción en estudiantes universitarios. Estudios Pedagógicos, XL/I (1), 255-269. http://doi.org/10.4067/S0718-07052016000100016

Sobrados, M. (2016). El trabajo docente en grupos numerosos. Experiencias en el uso del portafolio. Opción, 32(10), 773-788.

Webster, B. J., Chan, W. S. C., Prosser, M. T., y Watkins, D. A. (2009). Undergraduates' learning experience and learning process: quantitative evidence from the East. Higher Education, 58(3), 375-386. http://doi.org/10.1007/s10734-009-9200-6

Copyright @ 2018 . Esta obra está sujeta a una licencia de Creative Commons mediante la cual, cualquier explotación de ésta, deberá reconocer a sus autores, citados en la referencia recomendada que aparece al inicio de este documento.

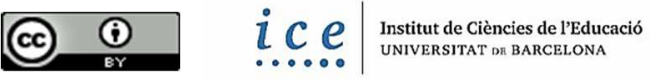

